\title{
A modular and flexible system for activity recognition and smart home control based on nonobtrusive sensors
}

\author{
Johannes Kropf*, Lukas Roedl*, Andreas Hochgatterer* \\ ${ }^{*}$ Health \& Environment Department, AIT Austrian Institute of Technology GmbH, Wiener Neustadt, Austria \\ Email: \{johannes.kropf, lukas.roedl, andreas.hochgatterer $\} @$ ait.ac.at
}

\begin{abstract}
This work describes a modular open source AAL framework for event recognition and smart home control. Various integrated tools simplify the configuration task, the personalization as well as the learning of activity models by a novel approach. Flexibility, standard compliant interfaces as well as the ability to transfer the system into new environments with little efforts have a strong focus. The paper describes the system architecture and the algorithms used.
\end{abstract}

\section{Motivation \& BACKGROUND}

The motivation of the activities and the work presented here are mainly driven by the demographic changes and the need for ambient assistive technologies for supporting a longer independent living of the elderly. Behavior pattern recognition based on nonobtrusive sensors is a challenging task but seen to be of fundamental importance for AAL technologies being accepted by the end users [1].

\section{A. State-of-the art in smart home technologies}

Future smart home solutions are demanded being able to increase the comfort and safety on the habitants and to save energy at the same time. Nowadays state-of-the-art smart home solutions are based on simple pre-defined rules to switch devices based on movement detectors or timers, but there is still very less intelligence built in due to the following reasons:

- Too many systems on the market are proprietary and cannot be connected to each other

- Very few smart appliances are available on the market (smart fridge, ...)

- Living environments differ significantly and standardized solutions will not work

- This leads to a very high configuration effort and high costs subsequently

The key towards smart systems is seen to be activity recognition and a lot of research was done over the last years. Even if many approaches and algorithms exist, there are still many problems to solve to gain a system which is able to be used in real environments in everyday life.

Besides the former focus on safety features for assistive enironments there has been increasing research in the area of activity and pattern recognition for diagnoses and therapy support. Sensor data is used for behavior monitoring and regarding trend analysis for early detection of neurodegenerative diseases (e.g. dementia) in several research projects. The project SOFTCARE for instance aims to develop a home monitoring system including the following features fall detection indoors and outdoors, behavior pattern recognition based on activity and location information and risk situations detection based on expert knowledge [2], [3]. A further German project with a diagnoses support goal is SAMDY [4] where a sensor-based adaptive monitoring system for behavior analysis for seniors is developed.

One of the first AAL Joint Programme projects in this area is the BEDMOND project where the authors are involved in and which aims at the development of an ICT-based system for an early detection of Alzheimer's disease and other neurodegenerative diseases, focused on elderly people while living at home [5], [6]. With such an early detection health professionals can later on apply an also early treatment which will help the elder to live longer in an independent way at home by delaying as long as possible Alzheimer's disease appearance. The project has started in June 2009, is now in its trial phase and will last until May 2012.

BEDMOND is based on a constant monitoring of the elders' behavior during their daily living so it can be continuously matched against a user profile set up within a training period. The results of this periodical matching can provide relevant information to the health experts to evaluate whether an Alzheimer's disease at early stage could appear to start. So, all the data gathered by the BEDMOND system, initially taken from home sensors network, later processed to daily activities recognition patterns and finally interpreted through a rulebased engine (where health professionals knowledge is the key), will be later, periodically presented to the medical expert to determine whether, at the sight of the reports, activity by activity, the behavior changes shown may mean the beginning of a cognitive decline or just a casual deviation. After detection, health experts will very likely apply a pharmacological treatment to the elderly person and the BEDMOND system will keep on monitoring user behavior in order to assure that the supported treatment takes effect on the delay of Alzheimer's disease appearance. 


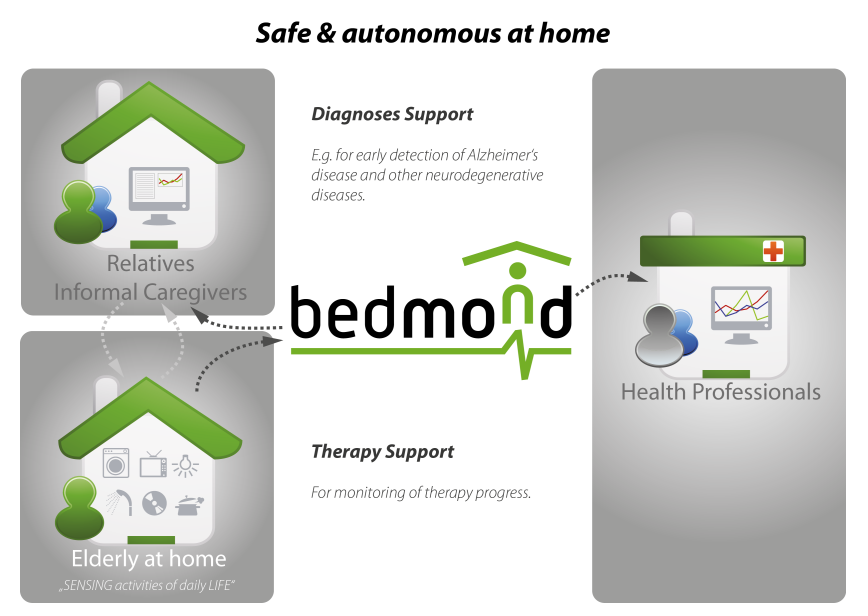

Fig. 1. The BEDMOND concept

Figure 1 shows the general concept of BEDMOND and the stakeholders addressed.

For a system being successful on the market it is essential to be flexible and easily configurable, what can be achieved only by using open and standardized interfaces to allow high interoperability with other systems. This work describes a system which is trying to overcome this difficulties in means of abstracted hardware layers and automated behavior model learning.

\section{B. Related Work}

Human activity recognition is a challenging task and has been an active, fast growing and huge research area and a multitude of approaches and algorithms have been proposed. Roughly, approaches can be categorized by vision-based, sensor-based, wearable or object based activity recognition, whereby this paper is focused on nonobtrusive sensor based activity recognition. Algorithms used in activity recognition can be divided into two major groups. The first one is based on machine learning techniques including supervised and unsupervised learning methods. The second one is based on logical modeling and reasoning [1].

For probabilistic activity recognition a wide range of algorithms exist. This includes Hidden Markov Models (HMMs) [7], Bayes networks [8], decision trees and other classical pattern recognition methods. Among these, HMMs and Bayes networks are the most popular used.

An approach similar to Hidden Markov Models are that of probabilistic finite state automata (PFSA) [9]. PFAs have been introduced to describe distributions over strings but have been successfully used in several fields, including pattern recognition. Finite state machines in general are widely used for all kind of control tasks, as they appear in smart environments as well.

Another approach for the detection of patterns in behavior are T-Patterns [10]. T-Patterns were created for finding hidden or nonobvious temporal patterns in behavior.

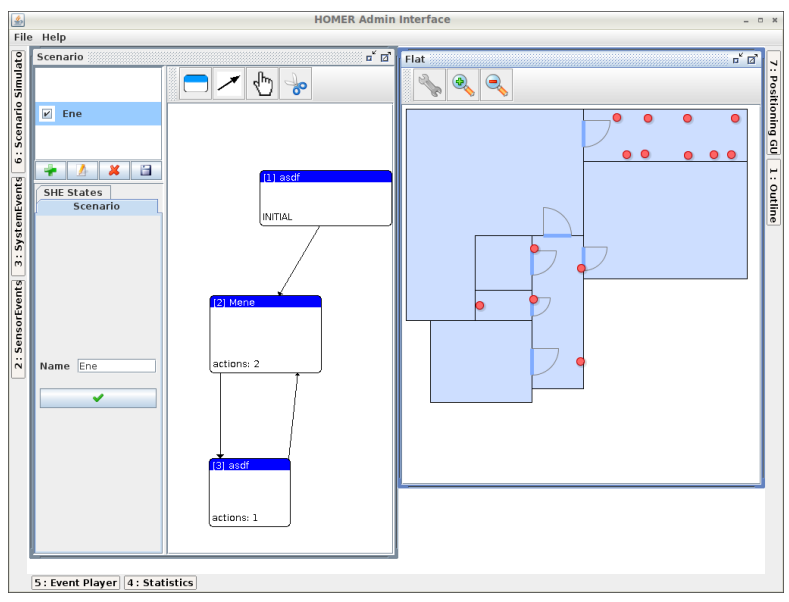

Fig. 2. Extendable HOMER administration interface

\section{The HOMe Event ReCognition System (HOMER)}

The HOMe Event Recognition System (HOMER) integrates the local (off-the-shelf) sensors and performs pre-processing. This open source platform [11] is based on an Apache Karaf OSGi framework and encapsulates its functionalities in terms of OSGi bundles which enables modularity. The bundles are executed on the Java Runtime Environment (JRE), which can be installed on various operating systems, what offers hardware independency. The usage of an OSGi framework provides remote maintenance and individual adaptability of the system. The components, coming in the form of bundles for deployment, can be remotely installed, started, stopped, updated and uninstalled without requiring a reboot of the system. Thus the framework is flexible in terms of expanding its functionality and updating single modules during runtime. The interactions and dependencies between bundles are handled by the framework itself. It manages searching and binding of required services, which are exposed functionalities within OSGi bundles, even when the service is activated at later time. Fine grained configuration options allow detailed access to functionalities in each OSGi bundle [12].

Along with OSGi several supporting technologies, like Apache Maven [13] and Eclipse Gemini Blueprint ( [14]; formerly known as Spring Dynamic Modules [15] which was migrated to the Eclipse foundation), are used. Standards for medical device communication and home automation networks are integrated to enable communication to appropriate devices. All of these technologies are used to realize important aspects for an AAL service platform: namely security, modularity, extendibility and interoperability.

Furthermore HOMER makes use of several standards, namely:

- Independent Living Activity Hub specialization ISO/IEEE 11073-10471.

- ISO/IEC 14543-3: KNX is a standardized OSI-based network communications protocol for intelligent buildings [16].

In the laboratory prototype typical off-the-shelf home au- 


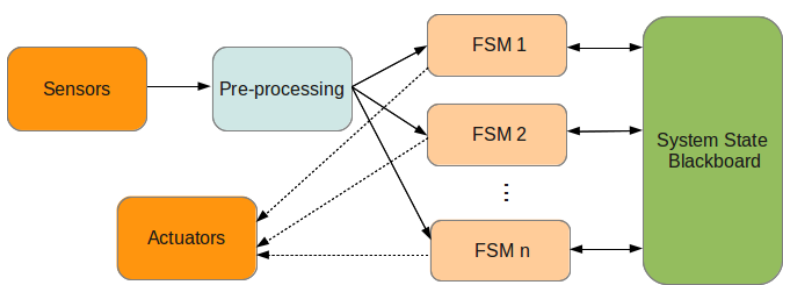

Fig. 3. HOMER finite state machine control system

tomation sensors, e.g. motion detectors, contact closure sensors and energy consumption sensors are used.

\section{A. Smart Home control}

The HOMER system is able to control devices within a smart home based on, up to now, finite state machine reasoning. Supported by a graphical user interface utility, the finite state machines can be configured easily and represented graphically, what allows also nonspecialists to configure the system. Even finite state machines have their shortcomings, they provide a good solutions for a lot of simple tasks which occur in a smart environments. Due to a global blackboard, conditions and consideration of also nonsensor events like global states which might get changed by another state machine, also complex dependencies between smart home states can be realized. The overall scheme is depicted in figure 3 .

With an appropriate pre-processing in means of detection outliers, incomplete or corrupted data, activity recognition can be performed with finite state machines. For example, good results could be achieved for the indoor localization task by reaching the second place at the EvAAL competition in 2011 [17]. In figure 2 the administration interface with a simple state machine is depicted.

\section{ACTIVITY RECOGNITION}

\section{A. Sensors integrated}

The sensors used in for behavior detection are off-the-shelf and low-cost conventional KNX home automation sensors and wireless xComfort sensors from EATON [18], which use a proprietary protocol, are applied and connected to HOMER. All sensor types have been integrated in the platform and are working in parallel due to the harmonization of data packets in a hardware abstraction bundle provided by HOMER. The passive infrared sensors (PIR) for motion detection and person tracking have been slightly adapted to decrease their detection area. Communication is established via RF-link to a gateway, which is USB-connected to a PC. All the sensors are battery powered and therefore do not need any cables, which eases the positioning and mounting in the home.

For ethical and acceptance reasons the project will not use video cameras and microphones and concentrate on unobtrusive sensor technology.

For the BEDMOND trials the 4 selected flats are located in the Elizaran Centre in San Sebastian (Spain), an assisted living facility, where older people live in an independent way but supported by a multidisciplinary team, according to their
TABLE I

INTEGRATED SENSORS

\begin{tabular}{|c|c|c|c|c|c|c|c|}
\hline & 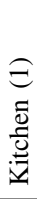 & 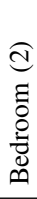 & 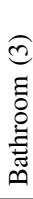 & 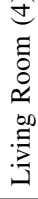 & 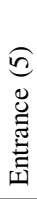 & $\begin{array}{l}\widehat{\theta} \\
\overline{0} \\
0 \\
0 \\
0\end{array}$ & \\
\hline reed switch & 4 & 7 & 0 & 0 & 1 & 1 & 13 \\
\hline power consumption & 0 & 1 & 1 & 2 & 0 & 0 & 4 \\
\hline pressure & 1 & 1 & 0 & 1 & 0 & 0 & 3 \\
\hline smoke sensor & 1 & 0 & 0 & 0 & 0 & 0 & 1 \\
\hline gas sensor & 1 & 0 & 0 & 0 & 0 & 0 & 1 \\
\hline water leak sensor & 1 & 0 & 0 & 0 & 0 & 0 & 1 \\
\hline \multirow[t]{2}{*}{ motion sensor } & 1 & 3 & 1 & 2 & 0 & 0 & 8 \\
\hline & 9 & 12 & 2 & 5 & 0 & 1 & \\
\hline
\end{tabular}

needs. Actually it has a day care centre for 20 olders, assisted living houses (10 apartments) and a shared housing setting. As an example the configuration of one of the selected flats is presented here. Figure 4 shows the floor plan with the locations of the used sensors. The table I lists all the integrated sensors.

\section{B. Pattern recognition (T-Patterns)}

The conceptual and algorithmic development behind TPatterns was initially stimulated by research regarding the structure of behavior and interactions with varying focus on real-time, probabilistic, and functional aspects, as well as hierarchical and syntactic structure, creativity, routines and planning [10], [19].

T-Patterns were chosen because behavior patterns are very often hidden in a behavioral stream and exist at highly different time scales. The T-Pattern method proposed in [10] is a simple approach to find patterns in time series data were causality between different data can be expected in short time intervals relative to the observation period. Even it was originally developed for studying of children's social interaction, it can be applied also to sensor data in smart environments where activity patterns are expected to be hidden in a data stream created by a large number of sensors. Additionally, as a probabilistic method the approach can handle noisy, uncertain and incomplete sensor data.

The T-Pattern approach is based on a null hypothesis, that each component is independently and randomly distributed over time with its observed average frequency. Making use of real-time information, the following probabilistic real-time relation serves as a key to the detection of hidden T-patterns. If $A$ is an earlier and $B$ a later component of the same recurring T-Pattern, then, after an occurrence of $A$ at $t$, there is an interval $\left[t+d_{1}, t+d_{2}\right],\left(d_{2} \geq d_{1} \geq 0\right)$ that tends to contain at least one occurrence of $B$ more often than it would be expected by chance. This relation is called a critical interval (or $\mathrm{CI}$ ) relation between the distributions of $A$ and $B$. The later component $B$ doesn't need to be the one immediately following $A$ within the pattern, so even when some elements of a T-Pattern are ignored, it may still have the defining 


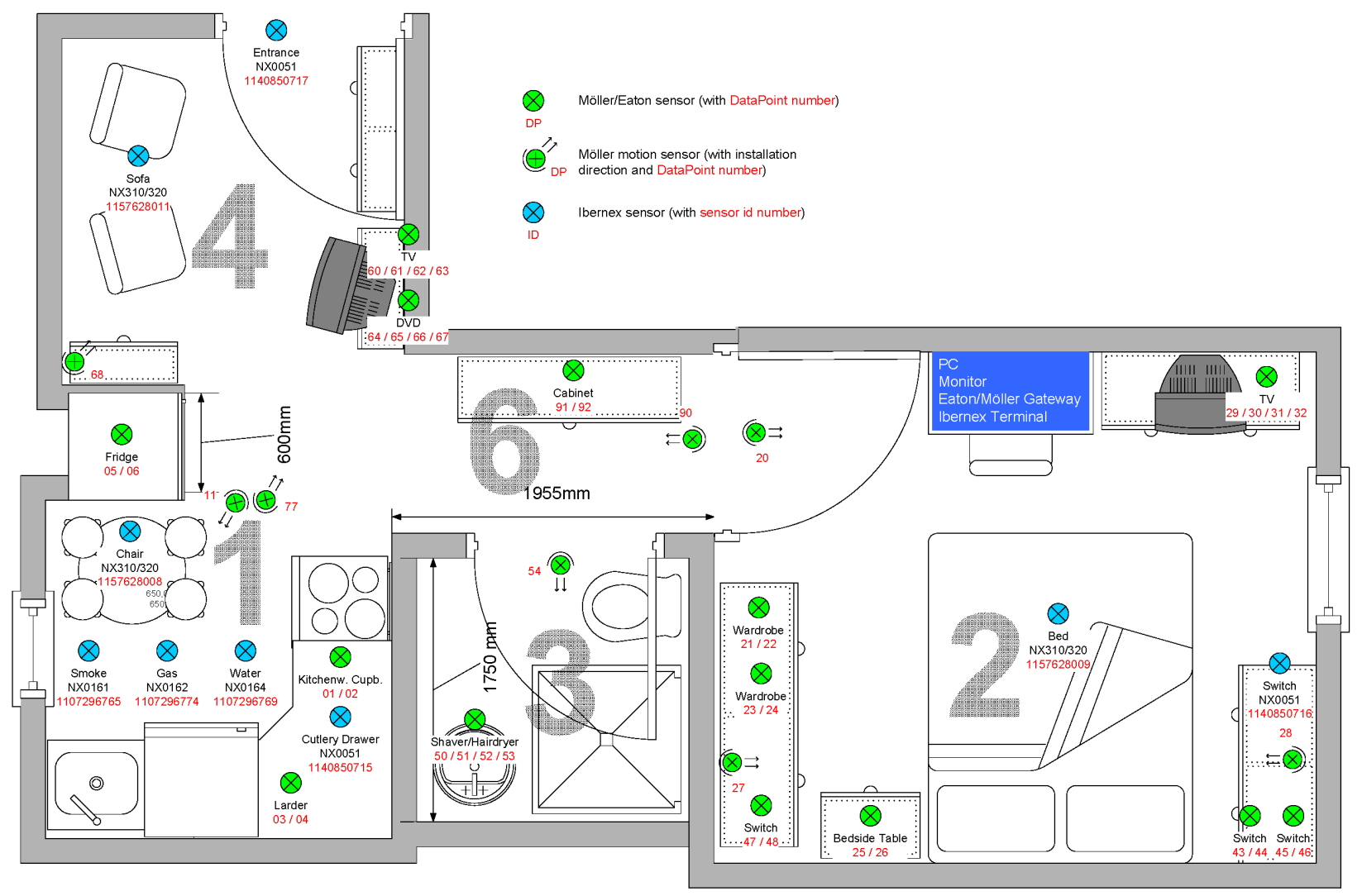

Fig. 4. Flat used in the BEDMOND trials with sensor locations

characteristics.

The behavior data are coded during an observation period $\left[1, N_{T}\right]$ in terms of discrete occurrence times of events. A TPattern is an ordered set $Q=X_{1} \approx d t_{1} X_{2} \approx d t_{2} \ldots X_{m}$, where $X$ stand for simpler T-Patterns and each $\approx d t$ term stands for a relatively invariant (relative to the expectation assuming the null hypothesis) time distance separating the consecutive $X_{i}$ and $X_{i+1}$ terms. The algorithm as proposed in [10] computes if there is a series of intervals containing $k(k \geq 2)$ occurrences of $A$ followed by at least on $B$ and checks if $k$ is significantly greater than the expectation by varying the interval length for the search.

When dealing with real world sensor data, the data are sparse in means of long observational periods and clustered in short periods. Figure 5 shows long periods without activity, and short periods with high activity. This must be considered when using the T-Pattern approach since the discrete observational time contributes to the computation of the significance value, what means that at first the discrete time points have to be normalized (e.g. to the shortest distance between to events to be one) and a very small significance level has to be chosen, otherwise long patterns will be recognized $\left(\alpha=10^{-8}\right)$.

A histogram of recognized T-Patterns based on 3 different sensors is depicted in figure 7. Several patterns appear significantly more often than others, and those can be assumed to be essential for an activity to be recognized. This detected patterns will found the basis with further processing when probabilistic state automata are going to be generated.

\section{Behavior model generation}

The T-Patterns detected with the procedure described in the previous section are the base for a further processing and activity model creation. For modeling a modified version of the algorithm for learning of variable memory length Markov processes is used as proposed in [20], [21]. In contrast to the original application in text recognition, where patterns consist of subsequent characterss, the patterns in our data might be noisy due to other sensor data which have been recorded in parallel but in another context. Since the noise might overweight the true patterns of interest, we see the TPattern approach as a preprocessing procedure to be a good method avoiding that disturbances.

Activity processes can be described by a subclass of probabilistic finite automata which are called Probabilistic Suffix Automata (PSA) by the authors of [20]. Probabilistic automata have similarities to widely used Hidden Markov Models, as analyzed e.g. in [22]. On the other hand finite state machines are already used in the HOMER [11] system and PSAs seemed to be a logical extension to handle also uncertainty in cases which can't be solved with pure finite state automata. 


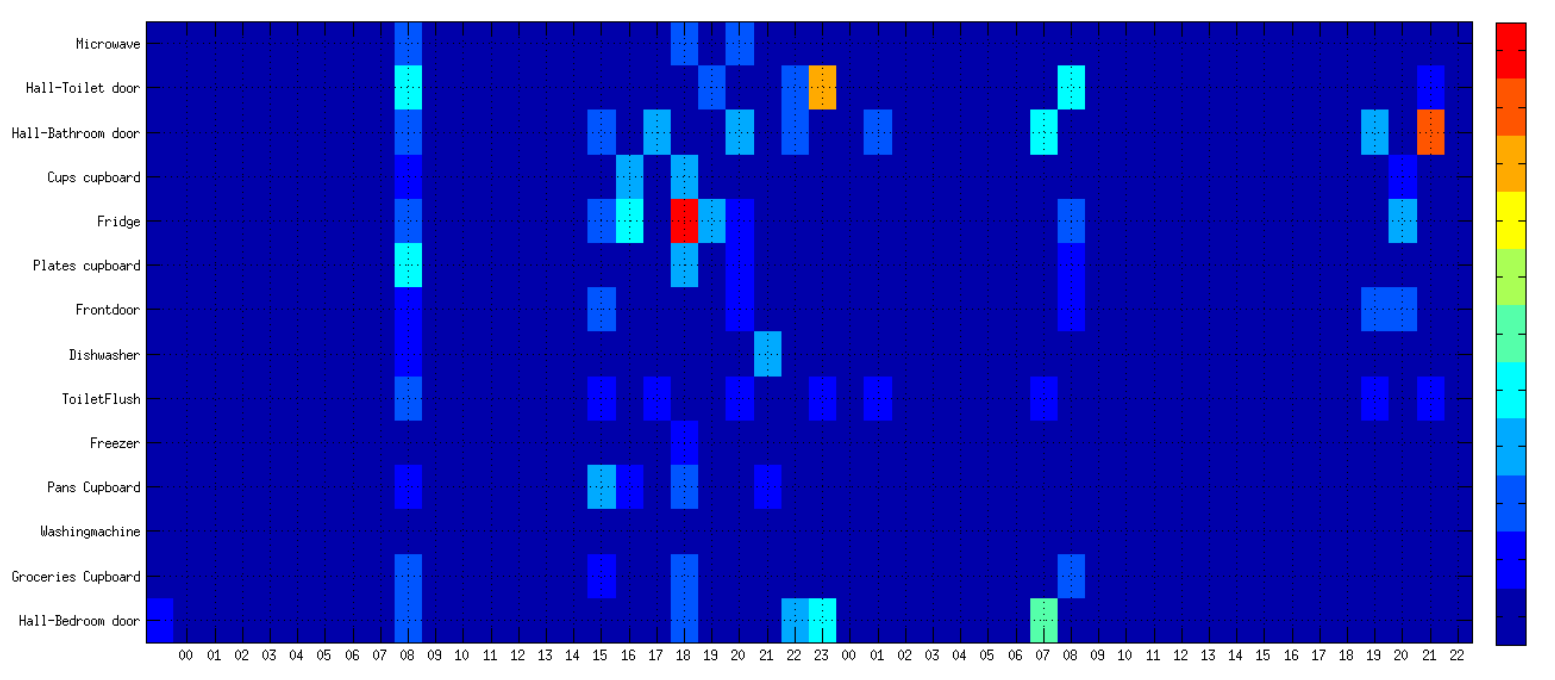

Fig. 5. Sensor data recorded 2 days, accumulated hourly

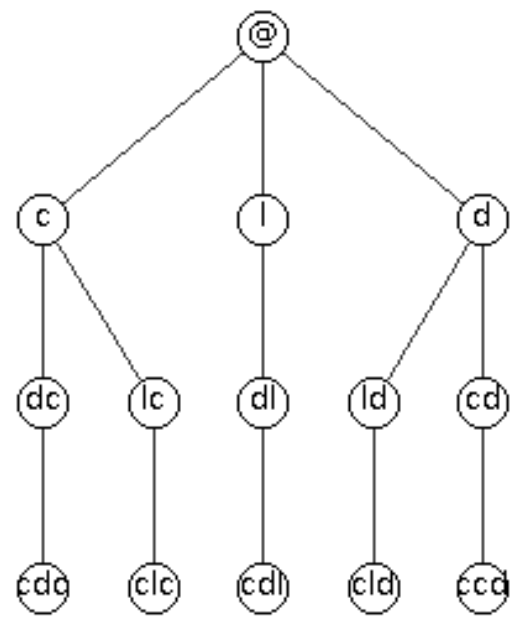

Fig. 6. PST generated analyzing patterns of 3 sensors, sensors coded as characters (c..toilet door, d..toilet flush, 1..front door)

In [20] the authors propose a learning algorithm using Prediction Suffix Trees which can be transformed to PSAs. It is shown that for every PSA there exists an equivalent PST of roughly the same size, but PSTs are easier to learn.

A PST $T$, over an alphabet $\Sigma$, is a tree of degree $|\Sigma|$. Each edge in the tree is labeled by a single symbol in $\Sigma$, such that from every internal node there is exactly one edge labeled by each symbol. the nodes of the tree are labeled by pairs $\left(s, \gamma_{s}\right)$ where $s$ is the string associated with the walk starting from that node and ending in the root of the tree, and $\gamma_{s}: \Sigma \rightarrow[0,1]$ is the next symbol probability function related with $s$. It is required that for every string s labeling a node in the tree, $\Sigma_{\sigma \in \Sigma} \gamma_{s}(\sigma)=1$. A PST $T$ generates strings of infinite length, but only probability distributions induced on finite length prefixes of these strings are considered. The probability that $T$ generates a string $r=r_{1} r_{2} \ldots r_{N} \in \Sigma^{N}$ is

$$
P_{T}^{N}(r)=\prod_{i=1}^{N} \gamma_{s^{i-1}}\left(r_{i}\right),
$$

where $s^{0}=e$, the empty string, and for $1 \leq j \leq N-1, s^{j}$ is the string labeling the deepest node reached by taking the walk corresponding to $r_{i} r_{i-1} \ldots r_{1}$ starting at the root of $T$.

The learning algorithm is as follows: The sample statistics is used to define the empirical probability function $\tilde{P}$ and a suffix tree $\bar{T}$ is constructed which is a subtree of $T$ with high probability. String with a single node labeled as the empty string $e$, nodes are added which are seen to be in the tree. This is the case when for the node $v$ the following holds. The empirical probability of $s, \tilde{P}(s)$, is non negligible, and for some symbol $\sigma, \tilde{P}(\sigma \mid s)$, differs substantially from the empirical probability of observing $\sigma$ following the suffix of s, which is $\tilde{P}(\sigma \mid \operatorname{suffix}(s))$. The decision rule is dependent on the ratio between $\tilde{P}(\sigma \mid s)$ and $\tilde{P}(\sigma \mid s u f f i x(s))$ and a given node is added only when this ratio is substantially greater than 1 . The detailed algorithm can be found in [20] as well as the transformation to a slight variant of a PSA. A learn PST based on real sensor data is shown in figure 6 .

\section{Detection of path}

The detection of repeating paths inside a home was one of the main indicators in the BEDMOND project [23], [24]. By using the T-Pattern recognition method, repeated path can be recognized and long period changes may indicate an increase of cognitive impairments. Due to the use of preprocessing the T-Pattern approach is very reliable here, especially because we use only certain events to find repeating pattern consisting of them. 


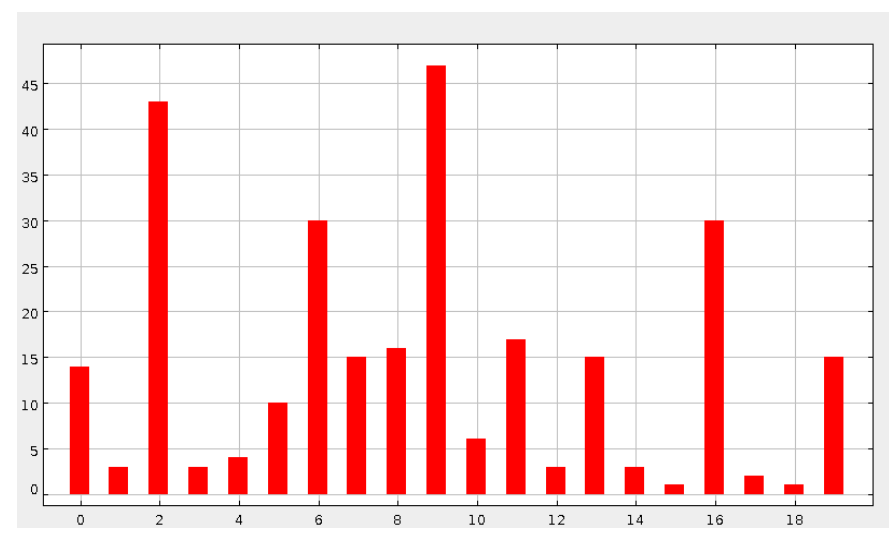

Fig. 7. Histogram of detected T-Patterns

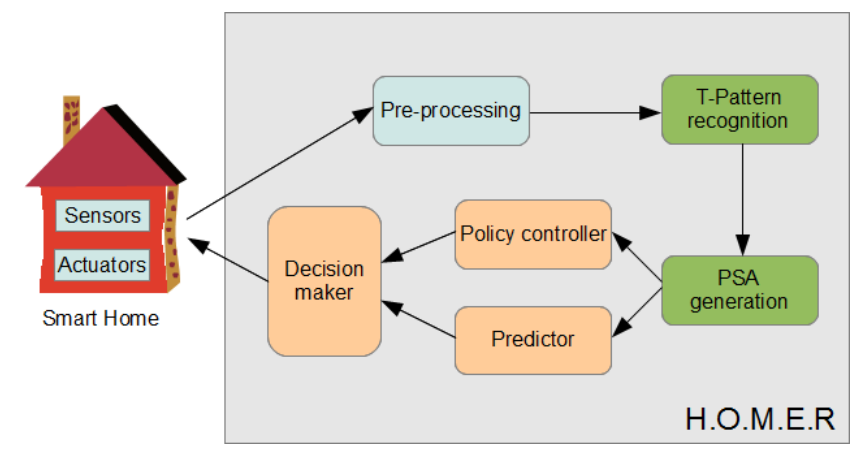

Fig. 8. HOMER activity recognition structure

\section{E. Detection of activity}

Activity recognition can be another simple modality for decision making tasks. The HOMER system simply measures the activity in means of active sensor times relative to a given time slot. For getting reliable results the data are checked for errors which are: incomplete data pairs (sensor usually send an $\mathrm{ON}$ and an OFF message), phantom messages (e.g. warm air streams detected by infrared sensors) and other. The algorithm gives a measurement of the overall activity for a given period per room or accumulated.

\section{F. Data acquisition}

Sensor data acquisition for pattern recognition in smart environments is always a big challenge since data are needed over a long period of time, especially when probabilistic methods are used. Much more challenging than the acquisition is the fact that annotated data are needed in order to train the models. Van Kasteren et al. [25] have provided an annotated nonobtrusive sensor based dataset over a period of approximately one month and three different smart environments. These dataset have been used in this work for pictures.

\section{CONCLUSION AND OUTLOOK}

The HOMER system is under current development and it will be extended in means of interoperability, pattern recognition methods and self-learning capabilities. The algorithms described beforehand will fundamentally increase the efficiency of the system in terms of configuration efforts due to automated processes. A conceptual structure of the system is depicted in figure 8. A strong impact on the further development have the outcomes of the universAAL project [26]. The universAAL project of the $7^{\text {th }}$ EU framework program aims to create an open and standard compliant reference platform to simplify and speed up an efficient development of home care and smart home services. The project is based on other former platforms and will be made available as open source especially for developers and service providers. The focus of the project is the increase of the acceptance of such systems and to provide technology for a wide range of stakeholders.

The core components of the HOMER system are available and will be developed further as open source under the Ambient Assisted Living Open Association (AALOA) [27].

The HOMER system aims to provide modules to the universAAL framework in means of administration interfaces and pattern recognition. To do so, the self-learning features of HOMER will be further extended and improved, and coupled with a context ware multi-device user interface system as under current development in den AALuis project [28].

\section{ACKNOWLEDGEMENT}

The authors wish to thank all the members of the BEDMOND Project Team, the ones close to end-users for their efficient work done while specifying requirements, the researchers highly involved in the Ambient Assisted Living environment and technologies to apply and, finally, the market oriented companies of the consortium which guide our development and thinking in the right way for the project results impact. This project is sponsored and partially funded by the European AAL Joint Programme and the National Funding Agencies from Austria, Portugal and Spain.

\section{REFERENCES}

[1] C. D. Nugent, J. Biswas, and J. Hoey, Activity Recognition in Pervasive Intelligent Environments (Google eBook). Springer, 2011. [Online]. Available: http://books.google.com/books?id=KKKdz7Ou8iUC $\backslash \&$ pgis= 1

[2] SOFTCARE project. (2012, Feb.). [Online]. Available: http://www. softcare-project.eu/

[3] K. Hofer and M. Höglinger, "User Needs in the SOFTCARE Project," Aging Clinical and Experimental Research, vol. 23, no. 1, p. 252, 2011.

[4] U. Gaden, E. Löhrke, M. Reich, W. Schöer, T. Stevens, and T. Vieregge, "SAMDY - Ein sensorbasiertes adaptives Monitoringsystem für die Verhaltensanalyse von Senioren," in Demographischer Wandel - Assistenzsysteme aus der Forschung in den Markt (AAL 2011). Beiträge des 4. Deutschen AAL-Kongresses, VDE, AAL, and BMBF, Eds., 2011.

[5] BEDMOND project. (2012, Feb.). [Online]. Available: http://www. bedmond.eu

[6] A. Hochgatterer, L. Roedl, A. Martinez, I. Etxeberria, E. Aldaz, B. Wöckl, and J. Bund, "Requirements for a Behaviour Pattern Based Assistant for Early Detection and Management of Neurodegenerative Diseases," in Proceedings of the PervasiveHealth Conference 2011, 2326 May 2011, Dublin, Published on CD-ROM, 2011.

[7] T. Mori, A. Fujii, M. Shimosaka, H. Noguchi, and T. Sato, "Typical Behavior Patterns Extraction and Anomaly Detection Algorithm Based on Accumulated Home Sensor Data," in Future Generation Communication and Networking (FGCN 2007). IEEE, 2007, pp.

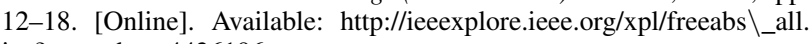
jsp?arnumber $=4426196$ 
[8] D. W. Albrecht, I. Zukerman, and A. E. Nicholson, "Bayesian Models for Keyhole Plan Recognition in an Adventure Game,' pp. 5-47, Mar. 1998. [Online]. Available: http://www.springerlink.com/content/ m34r486171345506/

[9] A. Paz, Introduction to Probabilistic Automata. New York: Academic Press, 1971.

[10] M. S. Magnusson, "Discovering hidden time patterns in behavior: T-patterns and their detection." Behavior research methods, instruments, \& computers : a journal of the Psychonomic Society, Inc, vol. 32, no. 1, pp. 93-110, Feb. 2000. [Online]. Available: http://www.ncbi.nlm. nih.gov/pubmed/10758668

[11] AIT Austrian Institute of Technology GmbH - Biomedical Systems. (2011, Mar.) HOMER - Home Event Recognition System. [Online]. Available: http://www.novahome.at/homer/entwickler-ressourcen/

[12] T. Fuxreiter, C. Mayer, S. Hanke, M. Gira, M. Sili, and J. Kropf, "A modular platform for event recognition in smart homes," in 2010 12th IEEE International Conference on e-Health Networking Applications and Services (Healthcom), 2010, pp. 1-6.

[13] Apache Maven Project. (2012, Feb.). [Online]. Available: http: //maven.apache.org

[14] Eclipse Gemini Blueprint. (2012, Feb.). [Online]. Available: http: //www.eclipse.org/gemini/blueprint/

[15] Spring Dynamic Modules for OSGi Service Platforms. (2012, Feb.) [Online]. Available: http://www.springsource.org/osgi

[16] KNX Association. (2012, Feb.). [Online]. Available: http://www.knx.org

[17] EvAAL competition 2011. (2012, Feb.). [Online]. Available: http: //evaal.aaloa.org/2011-competition/results

[18] Eaton Industries GmbH. (2012, Feb.) xComfort. [Online]. Available: http://www.moeller.net/en/products_solutions/power_distribution/ buildings/xcomfort

[19] M. S. Magnusson, "Repeated Patterns in Behavior and Other Biological Phenomena," Evolution of communication systems: A comparative approach, pp. 111-128, 2004.

[20] D. Ron, Y. Singer, and N. Tishby, "The Power of Amnesia : Learning Probabilistic Automata with Variable Memory Length," Machine Learning, vol. 25, no. 2, pp. 117-149, 1996.

[21] — , "The Power of Amnesia," Advances in Neural Information Processing Systems, vol. 6, pp. 176-183, 1993.

[22] P. Dupont, F. Denis, and Y. Esposito, "Links between probabilistic automata and hidden Markov models: probability distributions, learning models and induction algorithms," Pattern Recognition, vol. 38, no. 9, pp. 1349-1371, Sep. 2005. [Online]. Available: http://dx.doi.org/10.1016/j.patcog.2004.03.020

[23] E. Urdaneta, A. Iglesias, E. Aldaz, N. Galdona, A. Etxaniz, O. Subasi, L. Roedl, and A. Hochgatterer, "Early detection and monitoring of mild cognitive impairment through the register of daily behavior patterns," Alzheimer's and Dementia, vol. 6, no. 4, pp. e9-e9, Jul. 2010. [Online]. Available: http://linkinghub.elsevier.com/retrieve/ pii/S155252601002220X

[24] Bedmond Consortium. (2012, Feb.) BEDMOND. [Online]. Available: http://www.bedmond.eu

[25] T. L. van Kasteren, G. Englebienne, and B. J. A. Kröse, "Human Activity Recognition from Wireless Sensor Network Data: Benchmark and Software," in Atlantis Ambient and Pervasive Intelligence, L. Chen, C. D. Nugent, J. Biswas, and J. Hoey, Eds. Amsterdam: Atlantis Press, 2011, vol. 4, pp. 165-186.

[26] universAAL project. (2012, Feb.). [Online]. Available: http://www. universaal.org

[27] HOMER AALOA Project. (2012, Feb.). [Online]. Available: http: //homer.aaloa.org/

[28] AALuis project. (2012, Feb.). [Online]. Available: http://www.aaluis.org 